\title{
Analysis of Asymmetric Dual-Hop Energy Harvesting-Based Wireless Communication Systems in Mixed Fading Environments
}

\author{
Elmehdi Illi, Member, IEEE, Faissal El Bouanani, Senior Member, IEEE, Paschalis C. Sofotasios, Senior Member, \\ IEEE, Sami Muhaidat, Senior Member, IEEE, Daniel Benevides da Costa, Senior Member, IEEE, \\ Fouad Ayoub, Member, IEEE, and Ala Al-Fuqaha, Senior Member, IEEE
}

\begin{abstract}
In this paper, the performance of a dual-hop energy harvesting-based fixed-gain amplify-and-forward (AF) relaying communication system, subject to fading impairments, is investigated. We consider a source node $(S)$ communicating with a destination node $(D)$ through a fixed distant relay $(R)$, which harvests energy from its received signals and uses it to amplify and forward the received signals to $D$. Power-splitting (PS) and timeswitching (TS) schemes are considered in the analysis for energy harvesting. The $S-R$ and $R-D$ hops are modeled by the Nakagami$m$ and $\alpha-\mu$ fading models, respectively. Closed-form expressions for the statistical properties of the end-to-end signal-to-noise ratio (SNR) are derived, based on which novel closed-form expressions for the average symbol error rate (ASER) as well as average channel capacity (ACC) considering four adaptive transmission policies are derived. The derived expressions are validated through Monte-Carlo simulations.
\end{abstract}

Index Terms-Adaptive transmission policies, amplify-andforward, energy harvesting, mixed-fading environments.

\section{INTRODUCTION}

The ever-increasing demand for broadband communication systems and the growing number of connected devices are the driving forces for the evolution of wireless technologies in the past decades [1]. With the emergence of new paradigms such as the Internet of Things (IoT) as well as machineto-machine (M2M) communication, the next generation of wireless networks are envisioned to catalyze the deployment of new technologies and services, such as remote healthcare, surveillance, and transportation [2].

In addition to the need for higher data rates and expansion of network coverage, the maximization of the energy efficiency is among the most critical challenges for the fifth generation (5G) of wireless networks and beyond [3], where the power consumption and battery lifetime of wireless nodes are of paramount importance [1], [4]. Although energy resources (e.g., battery) are limited, the connected devices in 5G systems are envisaged to operate in multiple spectrum bands, as well as providing a real-time processing [5]. Additionally, M2M devices and wireless sensors are typically deployed in difficultto-reach areas, e.g., structural health monitoring and mine

E. Illi and F. El Bouanani are with ENSIAS College of Engineering, Mohammed V University, Rabat, Morocco (e-mails: \{elmehdi.illi, f.elbouanani\}@um5s.net.ma).

P. C. Sofotasios and S. Muhaidat are with the Center for Cyber-Physical Systems, Department of Electrical and Computer Engineering, Khalifa University, PO Box 127788, Abu Dhabi, UAE (e-mails: \{p.sofotasios, muhaidat\}@ieee.org).

D. B. da Costa is with the Department of Computer Engineering, Federa University of Ceará (UFC), Sobral-CE, Brazil (e-mail: danielbcosta@ieee.org)

F. Ayoub is with CRMEF, Kenitra, Morocco (e-mail: ayoub@crmefk.ma).

A. Al-Fuqaha is with the Department of Computer Science, Western Michigan University, Kalamazoo, MI 49008, USA (e-mail: ala.alfuqaha@wmich.edu). tunnels [5], [6], making the battery recharging or replacement impractical in most cases.

Recently, energy harvesting (EH) has emerged as an attractive solution that is envisioned to provide a greener and safer energy supply to self-sustainable wireless commutations [7]. Radio-frequency energy harvesting (RF-EH) was proposed recently as a promising solution to provide perpetual energy replenishment for wireless networks. RF-EH is realized by allowing wireless devices, equipped with dedicated $\mathrm{EH}$ circuits, to harvest energy from either ambient RF signals or dedicated RF sources [8], [9]. In M2M communications and wireless sensor networks (WSN), terminal nodes harvest energy from either access points or a dedicated power source/base stations [1]. RF-EH can be categorized into two main strategies, namely, wireless-powered communications (WPC) [10] and simultaneous wireless information and power transfer (SWIPT), which has been shown to provide noticeable gains in terms of power and spectral efficiencies by enabling simultaneous information processing and wireless power transfer [6]. In practical scenarios, it is difficult to perform information decoding and energy harvesting. Accordingly, two practical system designs, namely time-switching (TS), and power-splitting (PS), were proposed [6]. In the former, the receiver switches over time between information decoding and $\mathrm{EH}$, while in the latter, the received power is split into two streams, one for $\mathrm{EH}$ and the other for information processing [11].

On the other hand, RF communications are often impaired due to multipath fading and shadowing random phenomena, caused by the presence of reflectors, scatterers, and obstacles [12]. Within this context, several existing distributions were introduced, supported by field test measurements, to accurately describe the statistics of the signal variations due to fading, namely Rayleigh for a non-line of sight (NLOS)-based link, Rician for a LOS-based communication, as well as Nakagami$m$ and Weibull distributions for urban outdoor environments. In [13], $\alpha-\mu$ model was proposed as an alternative solution for modeling the fading amplitude in a mobile radio channel, since it includes a vast majority of the well-known fading distributions, namely Rayleigh, Weibull, and Nakagami- $m$, as special cases.

Multihop relaying, where information is communicated between two terminals (nodes) over multiple hops, has been extensively investigated in the literature. This multihop approach realizes several key advantages as compared to single-hop scenario, e.g., lower power consumption and better throughput. A variant of multihop relaying, known as cooperative diversity, or cooperative communications, has emerged recently as a promising approach to increase spectral and power efficien- 
cies, network coverage, and reduce outage probability, mostly used in infrastructure-less based networks. In cooperative communications, the relays process signals overheard from the source terminal and re-transmit them toward a destination. Two common relaying techniques are decode-and-forward (DF) and amplify-and-forward (AF) [14]. In DF relaying, the relay terminal decodes a received signal and then re-encodes it (possibly using a different codebook) for transmission to a destination. With AF relaying, the relay terminal re-transmits a scaled version of the received signal without any attempt to decode it [14]. Also, in scenarios, the cooperating nodes are typically located at different locations, and, therefore, asymmetric channels are often experienced [15]. Therefore, several research studies investigated the performance analysis of dual-hop transmission systems under asymmetric fading environments, for AF and DF relaying. Specifically, in [16], the authors investigated the performance of DF relaying systems in mixed fading environments, which were modeled by Rayleigh/Generalized-Gamma fading channels. The authors in [17] examined the performance of a dual-hop system in mixed fading environments subject to Rayleigh/Rician scenarios. In [18], the analysis was carried out assuming a dual-hop DF relaying protocol with HARQ retransmission scheme in Rayleigh/Rician environments, while the work in [15] dealt with the analysis of a full-duplex DF relaying system over generalized fading channels. Finally, the work in [19] focused on the analysis of a multiple-input multipleoutput (MIMO) relay network with dual-hop AF relaying, over asymmetric fading channels.

Within the context of $\mathrm{EH}$, the performance of multi-hop EH-based wireless communication systems has been widely investigated in the literature. In [20], the authors dealt with the bit error rate performance of a dual-hop EH-based system, by considering the AF and DF protocols and taking into account TS and PS, over Nakagami- $m$ fading channels. In [21], the performance of a dual-hop full-duplex EH-based system is analyzed, by considering both $\mathrm{AF}$ and DF protocols, while [22] dealt with the analysis of a multi-hop cognitive-radio EH-based network. The works in [9] and [23] analyzed the performance of a dual-hop multi-relay communication scheme and a MIMO dual-hop EH-based communication system, respectively.

Although the previous works added new insights, they have mainly focused on: outage probability, error rate, and capacity analysis. Furthermore, sporadic results have been reported on the performance of dual-hop EH-based communication systems subject to asymmetric fading conditions. To the best of the authors' knowledge, the performance analysis of asymmetric dual-hop EH-based communication systems, including the average symbol error rate (ASER) and the average channel capacity (ACC) over two distinct adaptive transmission policies, has not been addressed yet in the open literature. Owing to this fact, this work aims to fill this gap by investigating the ASER and capacity performance of EH-based dual-hop fixedgain AF relaying systems over four different adaptive transmit policies, namely, optimal power adaptation (ORA), optimal power and rate adaptation (OPRA), channel inversion with fixed rate (CIFR), and truncated channel inversion with fixed rate (TCIFR). In particular, asymmetric fading conditions are assumed over the two hops, with Nakagami- $m$ and $\alpha-\mu$ fading conditions being taken into account, for the first and second hops, respectively. Both TS and PS are considered in the analysis. Overall, the main contributions of this paper can be summarized as follows:

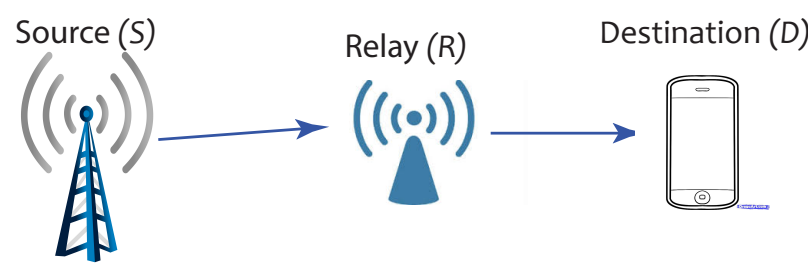

Fig. 1: System model

- Novel generalized closed-form expressions for the cumulative distribution function (CDF) and probability density function (PDF) of the end-to-end SNR of the considered system are derived, for both TS and PS schemes.

- Based on the statistical properties, novel closed-form expressions for the ASER for various modulation schemes and the ACC over four adaptive policies are derived.

The remainder of this paper is organized as follows. Section II describes the considered system and channel models, while Section III is dedicated to the statistical properties of the endto-end SNR, namely the CDF and PDF. Section IV focuses on the performance analysis of the considered system over various adaptive policies. Numerical results and discussions are presented in Section V, while conclusions are drawn in Section VI.

\section{Channel and System Models}

We consider a dual-hop EH-based wireless communication system with a fixed-gain AF relay, operating with both TS and PS schemes, as shown in Fig. 1 11 A source terminal $S$ communicates with the destination $D$ through a fixed relay node $R$ over two time slots. For the TS scheme, $S$ transmits the information signal to $R$ during the first part of the first time slot, and then transmits energy signal during the remaining part of it, while for PS, the power is divided at the relay into two portions, one for information decoding and the other for EH. The relay $R$ uses the energy harvested during Phase- 1 to amplify the received signal and forward it to $D$. It is assumed that no direct link exists between $S$ and $D$. We further assume asymmetric fading condition over the $S-R$ and $R-D$ hops. In particular, the $S-R$ link is subject to Nakagami-m fading channel, while the $R-D$ channel undergoes $\alpha-\mu$ fading.

\section{A. Phase I: $S-R$ hop}

\section{1) Information Signal Transmission:}

- TS protocol: The source node sends during the first $(1-$ $\varepsilon) T_{0}$ seconds the information signal $x_{s}$ to $R$, where $\varepsilon$ stands for the time portion dedicated to the $\mathrm{EH}$, and $T_{0}$ is the time slot dedicated to the $S-R$ communication. The received instantaneous SNR at the relay can be expressed as [12]

$$
\gamma_{1}^{(T S)}=\frac{P_{S}}{d_{1}^{\delta} N_{1}}\left|h_{1}\right|^{2}
$$

${ }^{1}$ Nakagami- $m$ fading model has been widely advocated in literature for providing a good agreement with urban land-mobile communication scenarios [12], [24]. On the other hand, $\alpha-\mu$ model can generalize fading scenarios for the same abovementioned environment, as well as for modeling ionospheric scintillation as shown in [25] and [26]. 
in which $P_{S}$ denotes the transmit power, $d_{R}$ is the $S$ $R$ distance, $\delta$ is the path-loss exponent, $h_{R}$ denotes the $S-R$ channel gain with average fading power $\Omega_{1}=$ $\mathbb{E}_{\left|h_{1}\right|}\left[\left|h_{1}\right|^{2}\right], \mathbb{E}_{X}[$.$] denotes the expected value with re-$ spect to the random variable $X$, and $N_{1}$ denotes additive white Gaussian noise (AWGN) power.

- PS protocol: $S$ sends during the first time slot $T_{0}$ the information signal $x$ with power $P_{S}$. The relay splits the power of the received signal into two parts: A portion $1-\varrho$ of the received power at the relay is dedicated to information decoding, where $0<\varrho<1$ is the power portion dedicated to EH. The received instantaneous SNR at the relay assuming PS scheme can be expressed as

$$
\gamma_{1}^{(P S)}=\frac{(1-\varrho) P_{S}}{d_{1}^{\delta} N_{1}}\left|h_{1}\right|^{2} .
$$

Consequently, the SNR $\gamma_{1}$ can be generalized for both schemes as follows

$$
\gamma_{1}=\frac{(1-\varsigma) P_{S}}{d_{1}^{\delta} N_{1}}\left|h_{1}\right|^{2} .
$$

with an average value

$$
\bar{\gamma}_{1}=\frac{(1-\varsigma) P_{S}}{d_{1}^{\delta} N_{1}} \Omega_{1},
$$

and $\varsigma$ equals either 0 for TS, and $\varrho$ for PS. In this work, Nakagami- $m$ fading model is considered for modelling the channel gain distribution of the $S-R$ hop. As a consequence, the received SNR at the relay is Gammadistributed with PDF/CDF expressions given as [12]

$$
\begin{aligned}
f_{\gamma_{1}}(z) & =\left(\frac{m_{1}}{\bar{\gamma}_{1}}\right)^{m} \frac{z^{m_{1}-1}}{\Gamma\left(m_{1}\right)} \exp \left(-\frac{m_{1}}{\bar{\gamma}_{1}} z\right), \\
F_{\gamma_{1}}(z) & =\frac{\gamma_{\text {inc }}\left(m_{1}, \frac{m_{1}}{\bar{\gamma}_{1}} z\right)}{\Gamma\left(m_{1}\right)},
\end{aligned}
$$

where $m_{1}$ stands for the Nakagami- $m$ fading parameter, $\Gamma($.$) and \gamma_{\text {inc }}(.,$.$) denote the Gamma and the$ lower-incomplete Gamma functions, respectively [27, Eqs. (8.310.1), (8.350.1)].

2) Energy Harvesting: For the TS scheme, the information is transmitted from $S$ to $D$ during a total time of $T_{0}$. Under this scheme, $\varepsilon$ represents the portion of $T_{0}$ in which the relay harvests energy from the source signal, where $0<\varepsilon<1$. Subsequently, data transmission is carried out in the remaining block transmission time. However, for the PS scheme, a portion $\varrho$ of the received power at the receiver is harvested by the relay. As a result, the collected energy by the relay for both schemes can be written as

$$
E_{R}=\frac{\theta \kappa T_{0} P_{S}\left|h_{1}\right|^{2}}{d_{1}^{\delta}},
$$

where $\kappa$ denotes $\varepsilon$ for TS and $\varrho$ for PS, and $\theta$ stands for the conversion efficiency of the relay's energy harvester.

The relay $R$ uses the harvested energy to amplify the received signal and to transmit it to $D$ during the time slot $T_{1}$. Henceforth, a finite battery storage model is assumed, where the harvested energy at the relay, $E_{R}$, can either be less than the relay battery capacity, or exceeds it. In the former case, the relay node communicates with $D$ with a power $P_{E}=\frac{E_{R}}{T_{1}}$, while in the latter one, the $R-D$ communication is ensured with the whole available power at the battery; i.e., $P_{B}=\frac{B_{R}}{T_{1}}$, with $B_{R}$ being the battery capacity in $\mathrm{mAh} \times \mathrm{V}$. Thus, the relay transmit power $P_{R}$ can be expressed as

$$
P_{R}=\left\{\begin{array}{l}
P_{E} ; \text { if } E_{R}<B_{R} \\
P_{B} ; \text { if } E_{R} \geq B_{R}
\end{array}\right.
$$

It can be easily shown that the PDF and CDF of $P_{E}$ are given by

$$
\begin{aligned}
f_{P_{E}}(y) & =\Psi^{m_{1}} \frac{y^{m_{1}-1}}{\Gamma\left(m_{1}\right)} \exp (-\Psi y), \\
F_{P_{E}}(y) & =\frac{\gamma_{\mathrm{inc}}\left(m_{1}, \Psi y\right)}{\Gamma\left(m_{1}\right)}
\end{aligned}
$$

where

$$
\Psi=\frac{m_{1} T_{1} d_{1}^{\delta}}{\theta \kappa T_{0} P_{s} \Omega_{1}}
$$

\section{B. Phase II: $R-D$ hop}

1) $E_{R}<B_{R}$ : During the second time slot $T_{1}$, the relay amplifies the signal received from $S$ by a fixed gain, and forwards it to $D$ using the harvested power $P_{E}$.

The received SNR at $D$ over the $R$ - $D$ link is given by

$$
\begin{aligned}
\gamma_{2}^{(1)} & =\frac{P_{E}\left|h_{2}\right|^{2}}{d_{2}^{\delta} N_{2}} \\
& =P_{E} \Upsilon_{2} .
\end{aligned}
$$

Here, the $R-D$ fading coefficient is assumed to follow an $\alpha-\mu$ distribution. Consequently, the SNR $\Upsilon_{2}$ is also $\alpha-\mu$ distributed, whose PDF is given by [13]

$$
f_{\Upsilon_{2}}(z)=\frac{\alpha_{2} \mu_{2}^{\mu_{2}}}{2 \Gamma\left(\mu_{2}\right) \bar{\Upsilon}_{2}}\left(\frac{z}{\bar{\Upsilon}_{2}}\right)^{\frac{\alpha_{2} \mu_{2}}{2}-1} e^{-\mu_{2}\left(\frac{z}{\Upsilon_{2}}\right)^{\frac{\alpha_{2}}{2}}}
$$

with average value $\bar{\Upsilon}_{2}=\frac{\Omega_{2}}{d_{2}^{\delta} N_{2}}$, where $\alpha_{2}$ and $\mu_{2}$ denote the two physical fading parameters that reflect the nonlinearity and clustering, respectively, and $\bar{\gamma}_{2}^{(1)}$ is the average $R-D$ SNR of this first case, given as

$$
\bar{\gamma}_{2}^{(1)}=\mathbb{E}_{P_{E}}\left[P_{E}\right] \bar{\Upsilon}_{2},
$$

where $\mathbb{E}_{P_{E}}\left[P_{E}\right]=\frac{\theta \kappa T_{0} P_{s} \Omega_{1}}{T_{1} d_{1}^{\delta}}$

2) $E_{R} \geq B_{R}$ : In this scenario, the relay $R$ forwards the information signal to $D$, after amplifying it with a fixed gain with power $P_{B}=\frac{B_{R}}{T_{1}}$. Consequently, the received SNR at $D$ is expressed as

$$
\gamma_{2}^{(2)}=P_{B} \Upsilon_{2}
$$

with an average value

$$
\bar{\gamma}_{2}^{(2)}=P_{B} \bar{\Upsilon}_{2}
$$

\section{STATISTICAL PROPERTIES}

In this section, the CDF expression in closed-form for the end-to-end SNR is derived.

Since the relay node performs fixed-gain AF relaying, the total received SNR at $D$ is expressed as [14]

$$
\gamma_{e q}=\frac{\gamma_{1} \gamma_{2}}{\gamma_{2}+C}
$$

with $C$ being a fixed-gain relaying constant. 


\section{A. Cumulative Distribution Function}

Proposition 1. The CDF expression of the end-to-end SNR for the considered system is expressed as

$$
F_{\gamma_{e q}}(z)=F_{\gamma_{e q}^{(1)}}(z) F_{P_{E}}\left(P_{B}\right)+F_{\gamma_{e q}^{(2)}}(z) F_{P_{E}}^{c}\left(P_{B}\right)
$$

with

$$
\gamma_{e q}^{(i)}=\frac{\gamma_{1} \gamma_{2}^{(i)}}{\gamma_{2}^{(i)}+C}
$$

$F_{X}^{c}($.$) is the complementary C D F$ of $X$, and

$$
\begin{aligned}
F_{\gamma_{e q}^{(1)}}(z) & =1-\frac{\alpha_{2}}{2 \Gamma\left(m_{1}\right) \Gamma\left(\mu_{2}\right)} e^{-\frac{m_{1}}{\bar{\gamma}_{1}} z} \sum_{n=0}^{m_{1}-1} \sum_{p=0}^{n}\left(\begin{array}{c}
n \\
p
\end{array}\right) \\
& \times \frac{\left(\frac{m_{1}}{\bar{\gamma}_{1}} z\right)^{n-p}}{n !} H_{0,3}^{3,0}\left(\mu_{2}\left(\frac{m_{1}^{2} C z}{\bar{\gamma}_{1} \bar{\gamma}_{2}^{(1)}}\right)^{\frac{\alpha_{2}}{2}} \begin{array}{c}
-;- \\
\Delta_{1} ;-
\end{array}\right),
\end{aligned}
$$

and

$$
\begin{aligned}
F_{\gamma_{e q}^{(2)}}(z) & =1-\frac{\alpha_{2}}{2 \Gamma\left(\mu_{2}\right)} e^{-\frac{m_{1}}{\bar{\gamma}_{1}} z} \sum_{n=0}^{m_{1}-1} \sum_{p=0}^{n}\left(\begin{array}{c}
n \\
p
\end{array}\right) \frac{\left(\frac{m_{1}}{\bar{\gamma}_{1}} z\right)^{n-p}}{n !} \\
& \times H_{0,2}^{2,0}\left(\mu_{2}\left(\frac{m_{1} C z}{\bar{\gamma}_{1} \bar{\gamma}_{2}^{(2)}}\right)^{\frac{\alpha_{2}}{2}} \mid \begin{array}{c}
-;- \\
\Delta_{2} ;-
\end{array}\right)
\end{aligned}
$$

where $H_{p, q}^{m, n}\left(z \mid \begin{array}{c}\left(a_{i}, A_{i}\right)_{i=1: p} \\ \left(b_{k}, B_{k}\right)_{k=1: q}\end{array}\right), \quad p \geq n, \quad$ and $q \geq m$, denotes the Fox's H-Function [28], $\Delta_{1}=\left(\mu_{2}, 1\right),\left(p, \frac{\alpha_{2}}{2}\right),\left(m_{1}+\mu_{2}\left(1-\frac{\alpha_{2}}{2}\right), \frac{\alpha_{2}}{2}\right)$, and $\Delta_{2}=\left(\mu_{2}, 1\right),\left(p, \frac{\alpha_{2}}{2}\right)$.

Proof: The proof is provided in Appendix A.

\section{B. Probability Density Function}

Corollary 1. The PDF of the end-to-end SNR of the considered system can be retrieved readily by differentiating (17) yielding

$$
\begin{aligned}
f_{\gamma_{e q}}(z) & =\frac{\alpha_{2} e^{-\frac{m_{1}}{\bar{\gamma}_{1}} z}}{2 \Gamma\left(\mu_{2}\right)} \sum_{n=0}^{m_{1}-1} \sum_{p=0}^{n}\left(\begin{array}{c}
n \\
p
\end{array}\right) \frac{\left(\frac{m_{1}}{\bar{\gamma}_{1}} z\right)^{n-p}}{n !} \\
& \times\left(\frac{F_{P_{E}}\left(P_{B}\right)}{\Gamma\left(m_{1}\right)} U_{1}+F_{P_{E}}^{c}\left(P_{B}\right) U_{2}\right)
\end{aligned}
$$

with

$U_{i}=\left[\begin{array}{c|c}\left(\frac{m_{1}}{\bar{\gamma}_{1}}-\frac{n-p}{z}\right) H_{0,3-i+1}^{3-i+1,0}\left(\begin{array}{c|c}\varkappa z^{\frac{\alpha_{2}}{2}} & -;- \\ \Delta_{i} ;-\end{array}\right) \\ -\frac{\alpha_{2}}{2 z} H_{1,4-i+1}^{3-i+1,1}\left(\begin{array}{l|l}\varkappa z^{\frac{\alpha_{2}}{2}} & (0,1) ;- \\ \Delta_{i} ;(1,1)\end{array}\right)\end{array}\right], i=1,2$ with $\varkappa=\mu_{2}\left(\frac{m_{1}^{2-i+1} C z}{\bar{\gamma}_{2}^{(i)} \bar{\gamma}_{1}}\right)^{\frac{\alpha_{2}}{2}}$.

Proof: The proof is provided in Appendix $B$.

\section{PERformance Evaluation}

In this section, closed-form expressions for the ASER for different modulation techniques, and the ACC of four adaptive transmission policies, namely ORA, OPRA, CIFR, and TCIFR, are derived.
Lemma 1. Let $x \geq 0$ be a positive real number. It follows that

$$
\begin{aligned}
\Xi(x) & =\int_{x}^{\infty} z^{\omega-1} e^{-\lambda z} H_{p, q}^{m, n}\left(a z^{v} \mid \begin{array}{c}
\left(a_{i}, A_{i}\right)_{j=1: p} \\
\left(b_{k}, B_{k}\right)_{k=1: q}
\end{array}\right) d z \\
& =\lambda^{-\omega} M_{p+1, q}^{m, n+1}\left(a \lambda^{-v} \mid \begin{array}{c}
w,\left(a_{i}, A_{i}, 0\right)_{i=2: p+1} \\
\left(b_{k}, B_{k}, 0\right)_{k=1: q}
\end{array}\right),
\end{aligned}
$$

where $w=(1-\omega, v ; \lambda x), M_{p, q}^{m, n}\left(z \mid \begin{array}{c}\left(a_{i}, A_{i}, \alpha_{i}\right)_{i=1: p} \\ \left(b_{k}, B_{k}, \beta_{k}\right)_{k=1: q}\end{array}\right)$ denotes the generalized incomplete-upper Fox's $H$-Function [29].

Proof: The proof is provided in Appendix $C$.

It is noteworthy that the Fox's $H$-Function and the generalized incomplete-upper Fox's $H$-Function can be implemented efficiently in most popular computer software, such as Matlab or Mathematica [29].

\section{A. Average Symbol Error Rate}

The ASER is a common performance metric for evaluating the communication reliability over fading channels. For a communication system subject to random fading, it is defined as the statistical average value of the instantaneous symbol error rate. For a variety of signaling/modulation techniques, the ASER is defined as

$$
\begin{aligned}
\bar{P}_{s e} & =\mathbb{E}_{\gamma}\left[P_{s e}(\gamma)\right] \\
& =\int_{0}^{\infty} P_{s e}(\gamma) f_{\gamma}(\gamma) d \gamma
\end{aligned}
$$

For various modulation types, the ASER is given by

$$
\bar{P}_{s e}=\rho \int_{0}^{\infty} \operatorname{erfc}(\sqrt{\gamma \theta}) f_{\gamma}(\gamma) d \gamma,
$$

where $\rho$ and $\theta$ are two modulation-dependant parameters [30], and erfc (.) stands for the complementary Gaussian error function [27, Eqs. (8.250.1, 8.250.4)].

Proposition 2. The ASER of the considered communication system for a variety of modulation schemes is expressed as

$$
\bar{P}_{s e}=\rho\left[\begin{array}{l}
1-\frac{\alpha_{2} \sqrt{\frac{\tau}{\sigma \pi}}}{2 \Gamma\left(m_{1}\right) \Gamma\left(\mu_{2}\right)} \sum_{n=0}^{m_{1}-1} \sum_{p=0}^{n}\left(\begin{array}{c}
n \\
p
\end{array}\right) \frac{\left(\frac{m_{1}}{\bar{\gamma}_{1} \sigma}\right)^{n-p}}{n !} \\
\times\left[F_{P_{E}}\left(P_{B}\right) V_{1}+F_{P_{E}}^{c}\left(P_{B}\right) \Gamma\left(m_{1}\right) V_{2}\right]
\end{array}\right],
$$

where

$$
\begin{gathered}
V_{i}=H_{1,3-i+1}^{3-i+1,1}\left(\varkappa \mid \begin{array}{c}
\left(p-n+\frac{1}{2}, \frac{\alpha_{2}}{2}\right) ;- \\
\Delta_{i} ;-
\end{array}\right), i=1,2, \\
\sigma=\frac{m_{1}}{\bar{\gamma}_{1}}+\tau .
\end{gathered}
$$

Proof: The ASER expression in 25 can be expressed using the $\mathrm{CDF}$ as

$$
\bar{P}_{s e}=\rho \int_{0}^{\infty} z^{\frac{-1}{2}} e^{-\tau z} F_{\gamma_{e q}}(\gamma) d \gamma .
$$

By involving the CDF in (17) into (29), and making use of the identity [28, Eq. (2.19)] alongside with some algebraic manipulations, (26) is attained. 


\section{B. Optimal rate adaptation policy}

The bandwidth-normalized ACC under constant transmission power, namely ORA policy, is often known as Shannon capacity. By definition, it is expressed as [12]

$$
\bar{C}_{\text {ORA }}=\int_{0}^{\infty} \log _{2}(1+z) f_{\gamma_{e q}}(z) d z
$$

Alternatively, it can be expressed in terms of the complementary CDF of the SNR as

$$
\bar{C}_{O R A}=\frac{1}{\ln (2)} \int_{0}^{\infty} \frac{F_{\gamma_{e q}}^{c}(z)}{1+z} d z .
$$

Proposition 3. The ACC under ORA policy of the considered AF dual-hop system is expressed in (32) at the top of the next page where $\Lambda_{k}=\left(\begin{array}{c}\left(-n+p, 1, \frac{\alpha_{2}}{2}\right) ;-:(0,1) ;-:-;- \\ -;-:(0,1) ;-: \Delta_{k} ;-\end{array}\right)_{k=1,2}$, $H_{p_{1}, q_{1} ; p_{2}, q_{2} ; p_{3}, q_{3}}^{0, n_{1}, m_{2}, n_{2} ; m_{3}}(x, y \mid \Xi), \quad$ with $\quad \Xi$

$\left(a_{i}, \alpha_{i} ; A_{i}\right)_{i=1: p_{1}} ;\left(c_{i}, C_{i}\right)_{i=1: p_{2}} ;\left(e_{i}, E_{i}\right)_{i=1: p_{3}}$

$\left(b_{k}, \beta_{k} ; B_{k}\right)_{k=1: q_{1}} ;\left(d_{k}, D_{k}\right)_{k=1: q_{2}} ;\left(f_{k}, F_{k}\right)_{k=1: q_{3}}$ the bivariate Fox's H-Function [31].

Proof: By involving (19) into (31), and with the help of [32, Eq. (07.34.03.0271.01)], we obtain

$$
\begin{aligned}
\bar{C}_{\text {ORA }} & =\frac{\alpha_{2}}{2 \ln (2) \Gamma\left(m_{1}\right) \Gamma\left(\mu_{2}\right)} \sum_{n=0}^{m_{1}-1} \sum_{p=0}^{n}\left(\begin{array}{c}
n \\
p
\end{array}\right) \frac{\left(\frac{m_{1}}{\bar{\gamma}_{1}}\right)^{n-p}}{n !} \\
& \times \int_{0}^{\infty} z^{n-p} e^{-\frac{m_{1}}{\bar{\gamma}_{1}} z} H_{1,1}^{1,1}\left(z \mid \begin{array}{c}
(0,1) ;- \\
(0,1) ;-
\end{array}\right) \\
& \times H_{0,3}^{3,0}\left(\mu_{2}\left(\frac{m_{1}^{2} C z}{\bar{\gamma}_{1} \bar{\gamma}_{2}^{(1)}}\right)^{\frac{\alpha_{2}}{2}} \mid \begin{array}{c}
-;- \\
\Delta_{1} ;-
\end{array}\right) d z .
\end{aligned}
$$

By making use of the identities [32, Eq. (07.34.03.0228.01)] and [31. Eq. (2.3)], and using the same aforementioned steps on (20), we obtain the closed-form expression for the ACC under ORA policy given in (32).

It is worth mentioning that the univariate and bivariate Fox's $H$-Function can be implemented efficiently in Matlab and Mathematica [30].

\section{Optimal power and rate adaptation policy}

In the context of EH-enabled communications, several works have dealt with optimal rate and/or power adaptation such as in [33], [34]. To this end, the bandwidth-normalized ACC under OPRA policy is defined as the capacity of a fading channel with the source transmit power being adapted to maximize the achievable end-to-end capacity. Mathematically, it is expressed as [35]

$$
\bar{C}_{\text {OPRA }}=\int_{\gamma^{*}}^{\infty} \log _{2}\left(\frac{z}{\gamma^{*}}\right) f_{\gamma_{e q}}(z) d z,
$$

which can be alternatively written as

$$
\bar{C}_{O P R A}=\frac{1}{\ln (2)} \int_{\gamma^{*}}^{\infty} \frac{1}{z} F_{\gamma_{e q}}^{c}(z) d z,
$$

where $\gamma^{*}$ is the optimal cutoff SNR, that is the zero of the following function [35]

$$
\begin{aligned}
g(x) & =\int_{x}^{\infty}\left(\frac{1}{x}-\frac{1}{z}\right) f_{\gamma_{e q}}(z) d z-1 \\
& =\frac{1}{x}\left(1-F_{\gamma_{e q}}(x)\right)-\mathbb{E}_{\gamma_{e q}}\left[\frac{1}{\gamma_{e q}}\right]_{x}-1,
\end{aligned}
$$

where

$$
\begin{aligned}
\mathbb{E}_{\gamma_{e q}}\left[\frac{1}{\gamma_{e q}}\right]_{x} & =\int_{x}^{\infty} \frac{1}{z} f_{\gamma_{e q}}(z) d z \\
& =\sum_{k=1}^{3} \sum_{i=1}^{2} T_{k}^{(i)}(x),
\end{aligned}
$$

and

$$
\begin{aligned}
T_{1}^{(i)}(x) & =\frac{\left(i-1+(-1)^{i+1} F_{P_{E}}\left(P_{B}\right)\right) \alpha_{2}}{2 \Gamma^{2-i}\left(m_{1}\right) \Gamma\left(\mu_{2}\right)} \sum_{n=0}^{m_{1}-1} \sum_{p=0}^{n}\left(\begin{array}{c}
n \\
p
\end{array}\right) \frac{1}{n !} \\
& \times M_{1,3-i+1}^{3-i+1,1}\left(\mu_{2}\left(\frac{m_{1}^{2-i} C}{\bar{\gamma}_{2}^{(i)}}\right)^{\frac{\alpha_{2}}{2}} \begin{array}{c}
\eta(x) ;- \\
\Phi_{i} ;-
\end{array}\right),
\end{aligned}
$$

$$
\begin{aligned}
T_{2}^{(i)}(x) & =-\frac{\left(i-1+(-1)^{i+1} F_{P_{E}}\left(P_{B}\right)\right) \alpha_{2}(n-p)}{2 \Gamma^{2-i}\left(m_{1}\right) \Gamma\left(\mu_{2}\right)} \sum_{n=0}^{m_{1}-1} \sum_{p=0}^{n} \\
& \times \frac{\left(\begin{array}{c}
n \\
p
\end{array}\right)}{n !} M_{1,3-i+1}^{3-i+1,1}\left(\mu_{2}\left(\frac{m_{1}^{2-i} C}{\bar{\gamma}_{2}^{(i)}}\right)^{\frac{\alpha_{2}}{2}} \mid \begin{array}{c}
\xi(x) ;- \\
\Phi_{i} ;-
\end{array}\right),
\end{aligned}
$$

$$
\begin{aligned}
T_{3}^{(i)}(x) & =-\frac{\left(i-1+(-1)^{i+1} F_{P_{E}}\left(P_{B}\right)\right) \alpha_{2}^{2}}{4 \Gamma^{2-i}\left(m_{1}\right) \Gamma\left(\mu_{2}\right)} \sum_{n=0}^{m_{1}-1} \sum_{p=0}^{n} \frac{\left(\begin{array}{c}
n \\
p
\end{array}\right)}{n !} \\
& \times M_{2,4-i+1}^{3-i+1,2}\left(\mu_{2}\left(\frac{m_{1}^{2-i} C}{\bar{\gamma}_{2}^{(i)}}\right)^{\frac{\alpha_{2}}{2}} \mid \begin{array}{c}
(0,1,1), \xi(x) ;- \\
\Phi_{i} ;(1,1,1)
\end{array}\right),
\end{aligned}
$$

with $\Phi_{1}=\left(\mu_{2}, 1,1\right),\left(m_{1}+\mu_{2}\left(1-\frac{\alpha_{2}}{2}\right), \frac{\alpha_{2}}{2}, 1\right),\left(p, \frac{\alpha_{2}}{2}, 1\right)$, $\Phi_{2}=\left(\mu_{2}, 1,1\right),\left(p, \frac{\alpha_{2}}{2}, 1\right), \eta(x)=\left(p-n, \frac{\alpha_{2}}{2}, \frac{m_{1}}{\bar{\gamma}_{1}} x\right)$, and $\xi(x)=\left(1+p-n, \frac{\alpha_{2}}{2}, \frac{m_{1}}{\bar{\gamma}_{1}} x\right)$.

Remark 1. One can notice clearly that by setting $x=0$ in (37), the moment of order $n=-1$ of $\gamma_{e q}$ is achieved. Consequently, the incomplete Fox's H-Functions in (38)-(40) become conventional Fox's H-Functions.

By differentiating (36) with respect to $x$, and computing its limits at 0 and $\infty$, one gets

$$
g^{\prime}(x)=-\frac{1}{x^{2}} F_{\gamma_{e q}}^{c}(x)<0,
$$

$$
\lim _{x \rightarrow 0} g(x)=\lim _{x \rightarrow 0} \int_{x}^{\infty}\left(\frac{1}{x}-\frac{1}{z}\right) f_{\gamma_{e q}}(z) d z-1=\infty,
$$

and

$\lim _{x \rightarrow \infty} g(x)=\lim _{x \rightarrow \infty} \int_{x}^{\infty}\left(\frac{1}{x}-\frac{1}{z}\right) f_{\gamma_{e q}}(z) d z-1=-1$.

Given the above, the zero value of 36 is unique. 


$$
\bar{C}_{\text {ORA }}=\frac{\alpha_{2} \bar{\gamma}_{1}}{2 \ln (2) \Gamma\left(\mu_{2}\right) m_{1}} \sum_{n=0}^{m_{1}-1} \sum_{p=0}^{n}\left(\begin{array}{c}
n \\
p
\end{array}\right) \frac{1}{n !}\left[\begin{array}{c}
\frac{F_{P_{E}}\left(P_{B}\right)}{\Gamma\left(m_{1}\right)} H_{1,0: 1,1: 0,3}^{0,1: 1,1: 0}\left(\frac{\bar{\gamma}_{1}}{m_{1}} ; \mu_{2}\left(\frac{m_{1} C}{\bar{\gamma}_{2}^{(1)}}\right)^{\frac{\alpha_{2}}{2}} \mid \Lambda_{1}\right) \\
+F_{P_{E}}^{c}\left(P_{B}\right) H_{1,0: 1,1: 0,2}^{0,1: 1,1: 0}\left(\frac{\bar{\gamma}_{1}}{m_{1}} ; \mu_{2}\left(\frac{C}{\bar{\gamma}_{2}^{(2)}}\right)^{\frac{\alpha_{2}}{2}} \mid \Lambda_{2}\right)
\end{array}\right] .
$$

By involving (17) into (35) and using Lemma 1 for $x=\gamma^{*}$, a closed-form expression for the capacity under OPRA policy can be derived as

$$
\begin{aligned}
\bar{C}_{O P R A}= & \frac{\alpha_{2}}{2 \ln (2) \Gamma\left(\mu_{2}\right)} \sum_{n=0}^{m_{1}-1} \sum_{p=0}^{n} \frac{1}{n !}\left(\begin{array}{c}
n \\
p
\end{array}\right) \\
\times & {\left[\begin{array}{cc}
\frac{F_{P_{E}}\left(P_{B}\right)}{\Gamma\left(m_{1}\right)} M_{1,3}^{3,1}\left(\mu_{2}\left(\frac{m_{1} C}{\bar{\gamma}_{2}^{(1)}}\right)^{\frac{\alpha_{2}}{2}}\right. & \left.\begin{array}{c}
\xi\left(\gamma^{*}\right) ;- \\
\Phi_{1} ;-
\end{array}\right) \\
+F_{P_{E}}^{c}\left(P_{B}\right) M_{1,2}^{2,1}\left(\mu_{2}\left(\frac{C}{\bar{\gamma}_{2}^{(2)}}\right)^{\frac{\alpha_{2}}{2}}\right. & \left.\begin{array}{c}
\xi\left(\gamma^{*}\right) ;- \\
\Phi_{2} ;-
\end{array}\right)
\end{array}\right.}
\end{aligned}
$$

\section{Channel inversion with fixed rate Policy}

The CIFR policy is an adaptive transmission policy which requires that the transmitter exploits the channel state information (CSI) of both hops (i.e., $S-R$ and $R$ - $D$ hops), so that a constant SNR is maintained at the receiver (i.e., it inverts the channel fading). Mathematically speaking, the bandwidthnormalized ACC under CIFR policy can be formulated as [35]

$$
\bar{C}_{C I F R}=\log _{2}\left(1+\frac{1}{\int_{0}^{\infty} \frac{f_{\gamma_{e q}(z)}}{z} d z}\right) .
$$

By setting $x=0$ in $377-40$, the integral $\int_{0}^{\infty} \frac{f_{\gamma_{e q}}(z)}{z} d z$ is attained. Thus, the ACC under CIFR policy can be expressed as

$$
\bar{C}_{C I F R}=\log _{2}\left(1+\frac{1}{\sum_{k=1}^{3} \sum_{i=1}^{2} T_{k}^{(i)}(0)}\right) \text {. }
$$

\section{E. Truncated channel inversion with fixed rate policy}

As in the above-mentioned CIFR policy, TCIFR policy consists of channel fading inversion only above a fixed cutoff $\gamma_{0}$. The ACC under this policy is defined as

$$
\bar{C}_{T C I F R}=\log _{2}\left(1+\frac{1}{\int_{\gamma_{0}}^{\infty} \frac{f_{\gamma_{e q}}(z)}{z} d z}\right)\left(1-F_{\gamma_{e q}}\left(\gamma_{0}\right)\right),
$$

As done with the CIFR policy in 45, by setting $x=\gamma_{0}$ in 37)- 40, the integral $\int_{\gamma_{0}}^{\infty} \frac{f_{\gamma_{e}}(z)}{z} d z$ is attained. Thus, the ACC under TCIFR policy can be expressed as

$$
\bar{C}_{T C I F R}=\left(1-F_{\gamma_{e q}}\left(\gamma_{0}\right)\right) \log _{2}\left(1+\frac{1}{\sum_{k=1}^{3} \sum_{i=1}^{2} T_{k}^{(i)}\left(\gamma_{0}\right)}\right) .
$$

It is noteworthy that (26), (32), (44), (46), and (48) are new, general and easy-to-evaluate analytically for the ASER and ACC subject to various transmit policies. We emphasize that such expressions can be served as a benchmark for future studies, in addition to filling the gap that still exists in the current technical literature.

\section{Numerical Results}

In this section, some illustrative numerical examples are depicted in order to highlight the effects of key system parameters on the obtained performance metrics. Without loss of generality, we set $m_{1}=3, \Omega_{1}=\Omega_{2}=5$, as the $S$ - $R$ hop fading parameter and average power, and $\alpha_{2}=2$, for the $R-D$ hop fading parameters. In addition, the relaying fixed-gain constant was set to $C=1$. Additionally, we set $T_{0}=T_{1}=1 \mathrm{~s}$, and $\varepsilon=\varrho=\theta=$ 0.7 , and $B_{R}=500 \mathrm{mAh} \times \mathrm{V}$ as the time slots in seconds, the harvester RF-to-DC conversion efficiency, the time slot ratio, and the battery capacity, respectively. Additionally, the complex contours of integration for computing (32) were chosen so as to separate left half-plane poles and right half-plane ones, for both integrand terms on $s$ and $t$, that are $\left.\mathcal{C}_{s}=\right] c_{s}-i \infty, c_{s}+i \infty[$ and $\left.\mathcal{C}_{t}=\right] c_{t}-i \infty, c_{t}+i \infty\left[\right.$, with $0<c_{s}<1$ and $c_{t}>\max \left(-\mu_{2},-\frac{2 k}{\alpha_{2}}, \frac{-2 \mu_{2}}{\alpha_{2}}\left(m_{1}+\mu_{2}\left(1-\frac{\alpha_{2}}{2}\right)\right)\right)$.

Figs. 2 and 3 depict the ASER performance vs the transmit power-to-noise ratio $\frac{P_{S}}{N_{1}}$ and the $S-R$ distance $d_{1}$. We consider TS scheme, with $\frac{P_{S}}{N_{2}}=100 \mathrm{~dB}, d_{2}=25 \mathrm{~m}, m_{1}=3$, and $\mu_{2}=4.2$, for various modulation schemes, based on 26. The analytical and simulation results match, which demonstrates the accuracy of the derived results. Additionally, it can be clearly seen that the ASER decreases significantly as a function of $\frac{P_{S}}{N_{1}}$. In fact, the greater the transmit power, the higher is the harvested energy, and consequently, the better is the bit error rate performance. That is, for $\frac{P_{S}}{N_{1}} \rightarrow \infty$, we converge to the performance of an AWGN channel (no fading). Furthermore, one can remark also from Fig. 2 2 the nodes distance effect on the ASER performance. The farther $S$ and $R$, the more severe the path loss, degrading the overall system's performance. Finally, the ASER performance result shows that BPSK modulation scheme results in a better error rate performance compared to its BFSK and QPSK counterparts.

Fig. 4 shows the ASER performance as a function of $\frac{P_{S}}{N_{1}}$, for several values of $\frac{P_{S}}{N_{2}}$. Similar to Figs. 2 and 3 , it can be noticed that the ASER increases significantly as a function of $\frac{P_{S}}{N_{1}}$. Interestingly, one can remark that above certain $\frac{P_{S}}{N_{2}}$ values (e.g., $40 \mathrm{~dB}$ ), for a given $\frac{P_{S}}{N_{1}}$ value, the ASER is not improved significantly. That is, the end-to-end ASER performance converges to that of the $S-R$ hop.

Fig. 5 shows the ACC performance under ORA and OPRA policies, which are given by (32) and (44), respectively, versus $\frac{P_{S}}{N_{1}}$, with $\frac{P_{S}}{N_{2}}=100 \mathrm{~dB}, d_{1}=d_{2}=30 \mathrm{~m}$, and $\mu_{2}=4.2$. The respective optimal SNR $\gamma^{*}$ for the OPRA policy was computed numerically using 36. Similarly to ASER, it can be evidently seen that the overall ACC improves by increasing $\frac{P_{S}}{N_{1}}$, that is either greater radiated power $P_{s}$ or lower noise power at the relay $N_{2}$. Additionally, the result confirms again the free space path-loss effect, due to the propagation distance, on the end-to-end system's performance. In fact, the greater the distance $d_{i}, i=1,2$, the lower the received end-to-end SNR, which results in degradation on the overall channel capacity. 


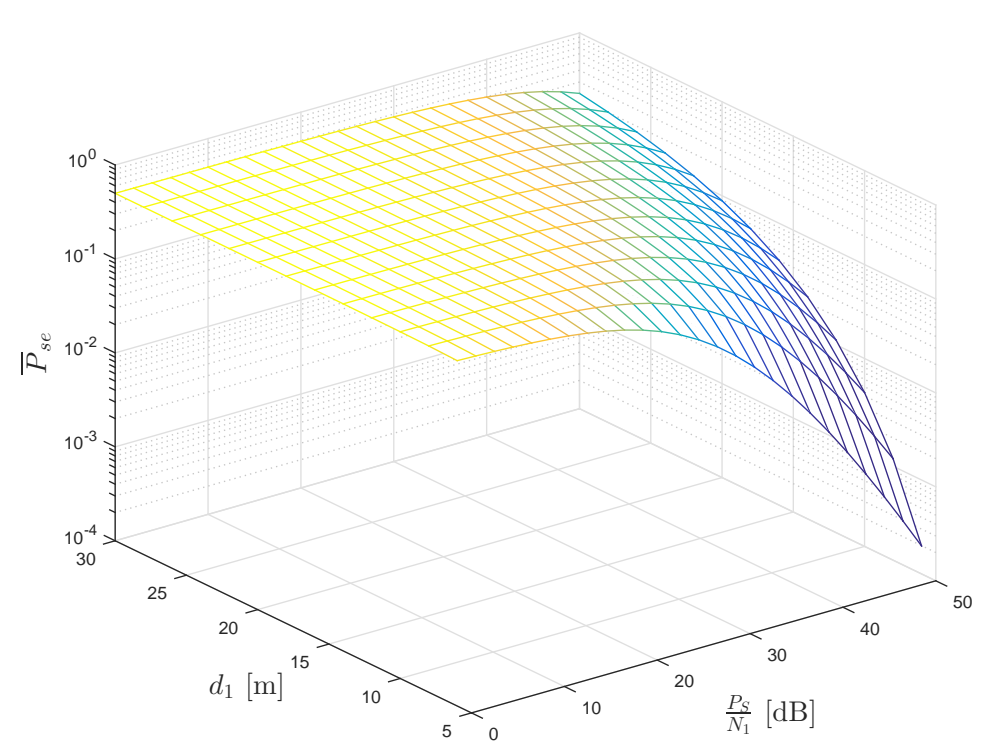

Fig. 2: ASER of the considered communication system for BPSK modulation versus $\frac{P_{S}}{N_{1}}$ and $d_{1}$.

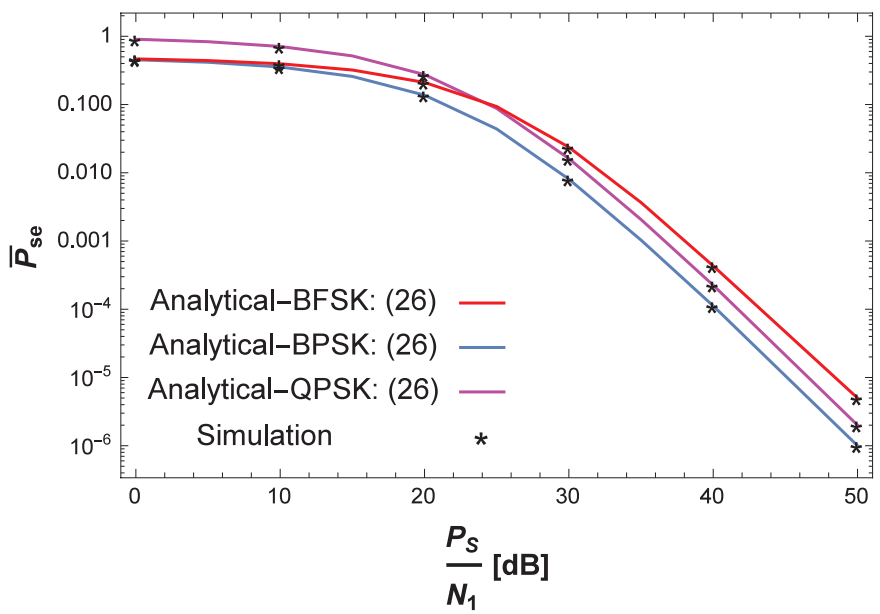

Fig. 3: ASER of the considered communication system versus $\frac{P_{S}}{N_{1}}$, for various modulation schemes.

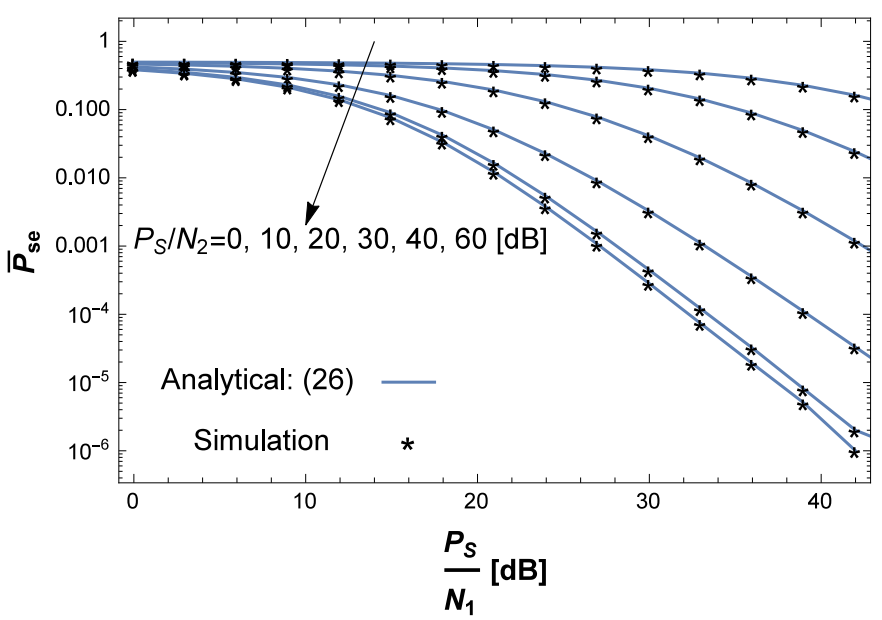

Fig. 4: ASER versus $\frac{P_{S}}{N_{1}}$ considering TS scheme, for several $\frac{P_{S}}{N_{2}}$ values.

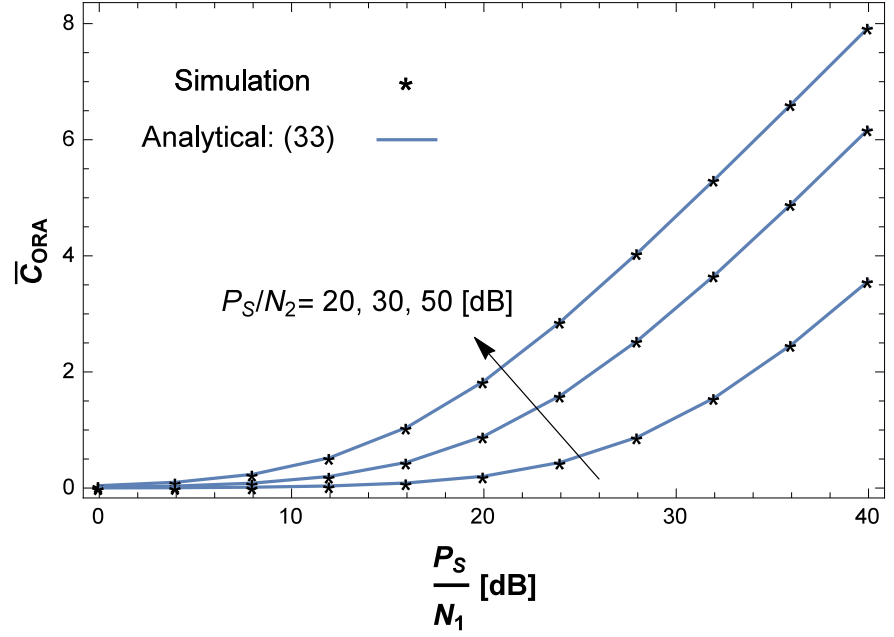

Fig. 5: ACC under ORA and OPRA policies of the considered communication system for TS scheme versus $\frac{P_{S}}{N_{1}}$.

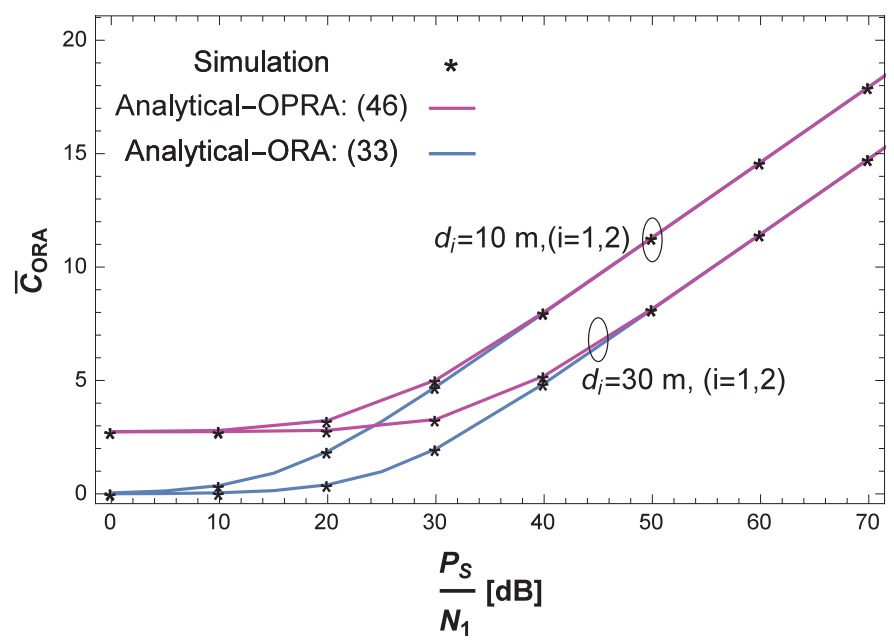

Fig. 6: ACC under ORA policiy of the considered communication system for TS scheme versus $\frac{P_{S}}{N_{1}}$, for various $\frac{P_{S}}{N_{2}}$ values.

Furthermore, it can be evidently seen that ACC under OPRA policy outperforms the ORA one, more particularly at high average SNR values.

In Fig. 6, the ACC under ORA policy is shown versus $\frac{P_{S}}{N_{1}}$ for various values of $\frac{P_{S}}{N_{2}}$, for $d_{i}=10 \mathrm{~m}$. Similarly to the $S-R$ power-to-noise ratio, the ACC increases also significantly by increasing the value $\frac{P_{S}}{N_{2}}$. Additionally, at higher values of $\frac{P_{S}}{N_{2}}$ (e.g., beyond $50 \mathrm{~dB}$ ), the ACC becomes steady for a given $\frac{P_{S}}{N_{1}}$ value. That is, the end-to-end SNR converges to the $S-R$ SNR, and consequently, the end-to-end ACC is that of the $S-R$ link.

In fig. 7, the ACC under ORA policy is shown versus the harvester efficiency $\varepsilon$ as well as the fading parameter $\mu_{2}$, for $\frac{P_{S}}{N_{0 i}}=20 \mathrm{~dB}, d_{i}=10 \mathrm{~m}, i=1,2$. One can ascertain that the ACC increases slightly as a function of the harvester efficiency $\varepsilon$. This shows that the greater $\varepsilon$, the higher the harvested power $P_{R}$, and consequently the relay can forward the information signal with a higher power. Interestingly, as we use TS protocol, even by increasing the duration dedicated to energy harvesting over information decoding one, the received SNR at $R$ is not impacted as can be seen from (1). In addition to this, the results show that the ACC is not impacted by the fading parameter $\mu_{2}$

Fig. 8highlights the ASER evolution versus the average SNR 


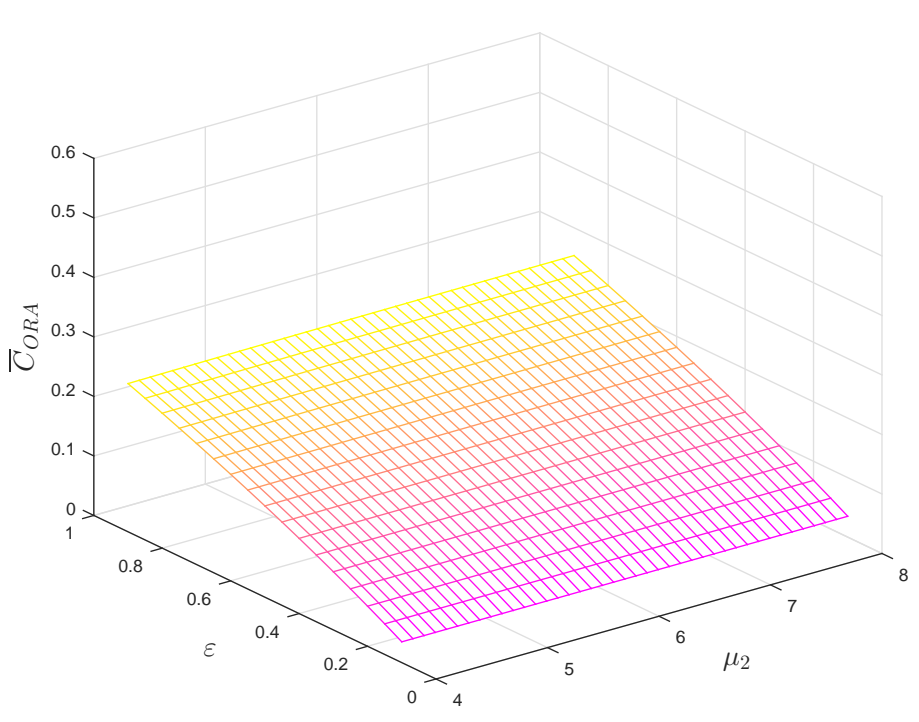

Fig. 7: ACC under ORA policy of the considered communication system for TS scheme versus $\varepsilon$ and $\mu_{2}$.

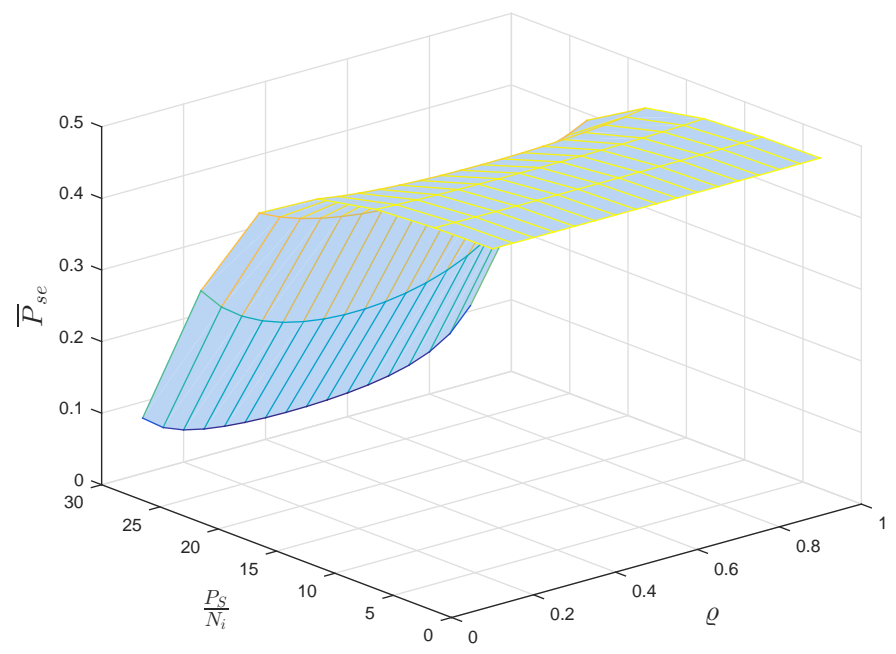

Fig. 8: ASER of the considered communication system for PS scheme versus $\varrho$ and $\frac{P_{S}}{N_{i}}, i=1,2$.

$\frac{P_{S}}{N_{i}}, i=1,2$, as well as the ratio $\varrho$, for PS scheme. Differently from the TS scheme, one can notice evidently that the ASER evolution versus $\varrho$ admits a minimum, particularly for high average SNR values, while for TS scheme as in fig. 7, the capacity increases as a function of $\varepsilon$. This can be explained from (1) and (3), where the SNR of the first hop for the PS scheme is affected by the ratio $\varrho$, whereas for its TS counterpart, the SNR $\gamma_{1}$ is not impacted by the ratio $\varepsilon$.

In Fig. 9, the ACC under CIFR and TCIFR policies is plotted versus the average SNR $\frac{P_{S}}{N_{1}}, i=1$, with $\frac{P_{s}}{N_{2}}=30 \mathrm{~dB}$, for TS and PS schemes. One can remark clearly that the ACC under CIFR policy surpasses slightly that under TCIFR policy, particularly for average SNR values that are less than $25 \mathrm{~dB}$. Nevertheless, this behavior is changed at high SNR values, where the TCIFR capacity slightly outperforms the CIFR one. In fact, in high average SNR regime, the end-to-end SNR is

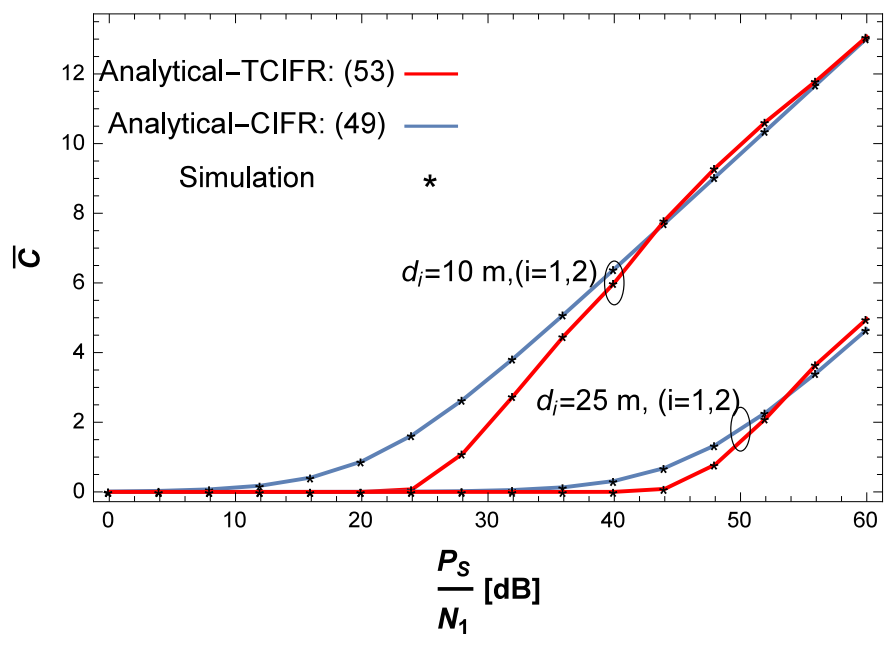

Fig. 9: ACC of the considered communication system under CIFR and TCIFR policies versus $\frac{P_{S}}{N_{1}}$, with $\frac{P_{S}}{N_{2}}=30 \mathrm{~dB}$.

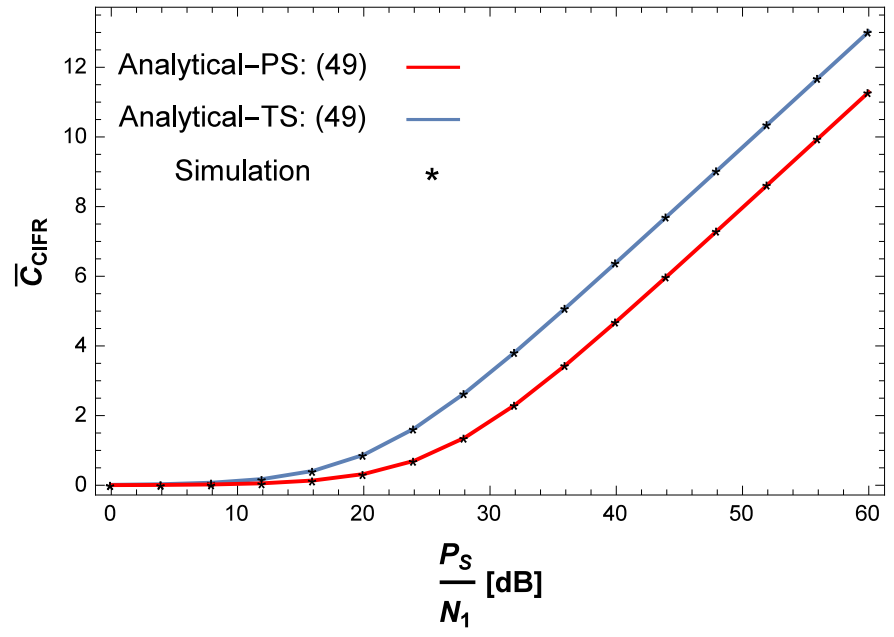

Fig. 10: ACC of the considered communication system under CIFR policy versus $\frac{P_{S}}{N_{1}}$, with $\frac{P_{S}}{N_{2}}=30 \mathrm{~dB}, d_{i}=10 \mathrm{~m}$, for TS and PS schemes.

relatively high. That is, the truncation of the channel inversion does not improve significantly the end-to-end channel capacity. Furthermore, the greater the $S-R$ and $R-D$ distance, the higher the free-space path loss.

The ACC under CIFR policy is plotted for both TS and PS schemes in Fig. 10. Interestingly, one can ascertain from the curves that the TS protocol clearly outperforms its PS counterpart in terms of capacity performance.

\section{CONCLUSION}

In this paper, a performance analysis of an AF dual-hop energy harvesting-based wireless communication system subject to asymmetric fading channels namely, Nakagami- $m$, and $\alpha-\mu$ was carried out. Both SWIPT schemes, namely power splitting and time switching, have been considered in the analysis. Statistical properties such as the CDF and PDF of the endto-end SNR were retrieved, in generalized expressions for both TS and PS schemes, based on which, closed-form expressions of the ASER as well as ACC over ORA, OPRA, CIFR, and TCIFR adaptive transmission policies have been derived. These analytical results were corroborated by Monte-Carlo simulation. 


\section{APPENDiX A: PRoOF of PROPOSITION 1}

Given the end-to-end SNR expression in (16), the respective $\mathrm{CDF}$ of $\gamma_{e q}$ is defined as [36]

$$
\begin{aligned}
F_{\gamma_{e q}}(z) & =\operatorname{Pr}\left[\gamma_{1}<z\left(1+\frac{C}{\gamma_{2}}\right)\right] \\
& =\int_{0}^{\infty} F_{\gamma_{1}}\left(z\left(1+\frac{C}{x}\right)\right) f_{\gamma_{2}}(x) d x
\end{aligned}
$$

Since $\gamma_{2}$ depends on the harvested power $P_{R}$, depending on whether $E_{R}<B_{R}$ or $E_{R} \geq B_{R}$, the CDF of the end-to-end SNR can be expressed as

$$
\begin{aligned}
F_{\gamma_{e q}}(z)= & F_{\gamma_{e q}}\left(z \mid P_{E}<P_{B}\right) \operatorname{Pr}\left[P_{E}<P_{B}\right] \\
& +F_{\gamma_{e q}}\left(z \mid P_{E} \geq P_{B}\right) \operatorname{Pr}\left[P_{E} \geq P_{B}\right]
\end{aligned}
$$

The conditional PDF of $\gamma_{e q}$ in each term of the above equation is expressed as

$$
F_{\gamma_{e q}}\left(z \mid P_{E}<P_{B}\right)=\underbrace{\int_{0}^{\infty} F_{\gamma_{1}}\left(z\left(1+\frac{C}{x}\right)\right) f_{\gamma_{2}^{(1)}}(x) d x}_{F_{\gamma_{e q}^{(1)}}(z)},
$$

$$
F_{\gamma_{e q}}\left(z \mid P_{E} \geq P_{B}\right)=\underbrace{\int_{0}^{\infty} F_{\gamma_{1}}\left(z\left(1+\frac{C}{x}\right)\right) f_{\gamma_{2}^{(2)}}(x) d x}_{F_{\gamma_{e q}^{(2)}}^{(z)}}
$$

One can note that evaluating (51) and $(52)$ corresponds to two distinct cases, namely $E_{R}<B_{R}$ and $E_{R} \geq B_{R}$, respectively.

- First case: $E_{R}<B_{R}$

One can notice from $\sqrt{12}$ that the $\gamma_{2}^{(1)}$ is the product of two random variables; namely $P_{E}$ and $\Upsilon_{2}$. Consequently, its PDF is expressed as

$$
f_{\gamma_{2}^{(1)}}(z)=\int_{0}^{\infty} \frac{1}{t} f_{P_{E}}\left(\frac{z}{t}\right) f_{\Upsilon_{2}}(t) d t
$$

By involving (9) and (13) into the above equation, as well as using the identity Eq. (07.34.03.0228.01) of [32] alongside with some algebraic manipulations, one obtains

$$
\begin{aligned}
f_{\gamma_{2}^{(1)}}(z) & =\frac{\alpha_{2} \mu_{2}^{\mu_{2}} \bar{\gamma}_{2}^{(1)}\left(\frac{\Psi z}{\bar{\gamma}_{2}^{(1)}}\right)^{\frac{\alpha_{2} \mu_{2}}{2}-1}}{2 \Gamma\left(m_{1}\right) \Gamma\left(\mu_{2}\right) z} \int_{0}^{\infty} G_{0,1}^{1,0}\left(\frac{\Psi z}{t} \mid \begin{array}{c}
-;- \\
\omega ;-
\end{array}\right) \\
& \times G_{0,1}^{1,0}\left(\mu_{2}\left(\frac{t}{\bar{\Upsilon}_{2}}\right)^{\frac{\alpha_{2}}{2}} \mid \begin{array}{c}
-;- \\
0 ;-
\end{array}\right) d t
\end{aligned}
$$

where $G_{p, q}^{m, n}(. \mid$.$) is the Meijer's G$-function [32, Eq. (07.34.02.0001.01)], $\Psi$ is defined in (11), and $\omega=m_{1}+$ $1-\frac{\alpha_{2} \mu_{2}}{2}$. Furthermore, using [32. Eqs. (07.34.16.0002.01, 07.34.21.0012.01)] and with some further manipulations, 54 can be expressed by

$f_{\gamma_{2}^{(1)}}(z)=\frac{\alpha_{2}}{2 z \Gamma\left(m_{1}\right) \Gamma\left(\mu_{2}\right)} H_{0,2}^{2,0}\left(\mu_{2}\left(\frac{m_{1} z}{\bar{\gamma}_{2}^{(1)}}\right)^{\frac{\alpha_{2}}{2}} \mid \begin{array}{l}-;- \\ \Upsilon ;-\end{array}\right)$.

with $\Upsilon=\left(\mu_{2}, 1\right),\left(m_{1}+\mu_{2}\left(1-\frac{\alpha_{2}}{2}\right), \frac{\alpha_{2}}{2}\right)$.
Substituting (55) and (6) into (49), and using the finite sum representation of the lower incomplete gamma function in [27 Eq. (8.352.1)] as well as [37. Eq. (2.9.4)] yields

$$
\begin{aligned}
F_{\gamma_{e q}^{(1)}}(z)= & 1-\frac{\alpha_{2} e^{-\frac{m_{1}}{\bar{\gamma}_{1}} z}}{2 \Gamma\left(m_{1}\right) \Gamma\left(\mu_{2}\right)} \sum_{n=0}^{m_{1}-1} \sum_{p=0}^{n}\left(\begin{array}{c}
n \\
p
\end{array}\right) \frac{\left(\frac{m_{1}}{\bar{\gamma}_{1}} z\right)^{n-p}}{n !} \\
& \times \int_{0}^{\infty} x^{-1} H_{0,1}^{1,0}\left(\frac{m_{1} C z}{\bar{\gamma}_{1} x} \mid \begin{array}{c}
-;- \\
(p, 1) ;-
\end{array}\right) \\
& \times H_{0,2}^{2,0}\left(\mu_{2}\left(\frac{m_{1} x}{\bar{\gamma}_{2}^{(1)}}\right)^{\frac{\alpha_{2}}{2}} \mid \begin{array}{c}
-;- \\
\Upsilon ;
\end{array}\right) d x
\end{aligned}
$$

Finally, by applying [37, Eqs. $(2.1 .3,2.8 .4)]$, [19] is attained.

- Second case: $E_{R} \geq B_{R}$

In this scenario, the SNR $\gamma_{2}$ is expressed as in (15). Hence, its respective PDF can be derived from (13) using [37, Eq. (2.9.4)] as

$$
\begin{aligned}
f_{\gamma_{2}^{(2)}}(z) & =\frac{\alpha_{2} \mu_{2}^{\mu_{2}}}{2 \Gamma\left(\mu_{2}\right) \bar{\gamma}_{2}^{(2)}}\left(\frac{z}{\bar{\gamma}_{2}^{(2)}}\right)^{\frac{\alpha_{2} \mu_{2}}{2}-1} e^{-\mu_{2}\left(\frac{z}{\bar{\gamma}_{2}^{(2)}}\right)^{\frac{\alpha_{2}}{2}}}, \\
& =\frac{\alpha_{2}}{2 \Gamma\left(\mu_{2}\right) z} H_{0,1}^{1,0}\left(\mu_{2}\left(\frac{z}{\bar{\gamma}_{2}^{(2)}}\right)^{\frac{\alpha_{2}}{2}} \mid \begin{array}{c}
-;- \\
\left(\mu_{2}, 1\right) ;-
\end{array}\right) .
\end{aligned}
$$

Similarly to $F_{\gamma_{e q}}^{(1)}(z)$, by involving the PDF of $\gamma_{2}$ above and (6) into (49), and using [27, Eq. (8.352.1)] as well as [37, Eq. (2.9.4)] we obtain

$$
\begin{aligned}
F_{\gamma_{e q}^{(2)}}(z) & =1-\frac{\alpha_{2}}{2 \Gamma\left(\mu_{2}\right)} e^{-\frac{m_{1}}{\bar{\gamma}_{1}} z} \sum_{n=0}^{m_{1}-1} \sum_{p=0}^{n}\left(\begin{array}{c}
n \\
p
\end{array}\right) \frac{\left(\frac{m_{1}}{\bar{\gamma}_{1}} z\right)^{n-p}}{n !} \\
& \times \int_{0}^{\infty} x^{-1} H_{1,0}^{0,1}\left(\frac{\bar{\gamma}_{1} x}{m_{1} C z} \mid \begin{array}{c}
(1-p, 1) ;- \\
-;-
\end{array}\right) \\
& \times H_{0,1}^{1,0}\left(\mu_{2}\left(\frac{x}{\bar{\gamma}_{2}^{(2)}}\right)^{\frac{\alpha_{2}}{2}} \mid \begin{array}{c}
-;- \\
\left(\mu_{2}, 1\right) ;-
\end{array}\right) d x
\end{aligned}
$$

By applying [37, Eqs. (2.1.3, 2.8.4)], 20) is obtained.

\section{APPENDIX B: PROOF OF COROLlaRY 1}

One can see that differentiating (17) with respect to $z$ yields

$$
f_{\gamma_{e q}}(z)=f_{\gamma_{e q}^{(1)}}(z) F_{P_{E}}\left(P_{B}\right)+f_{\gamma_{e q}^{(2)}}(z) F_{P_{E}}^{c}\left(P_{B}\right),
$$

By applying the derivative with respect to $z$ to $(19)$, one obtains (61) at the top of the next page, where $\Delta_{1}$ is defined in Proposition 1. Using the definition of Fox's $H$-Function in [38. Eqs. (1.5-1.6)] and given that $-s=\frac{\Gamma(1-s)}{\Gamma(-s)}$, by applying the derivative with respect to $z$, one gets

$$
\begin{aligned}
\mathcal{J} & =-\frac{1}{2 \pi j} \frac{\alpha_{2}}{2 z} \int_{\mathcal{C}_{s}} \Gamma\left(\mu_{2}+s\right) \Gamma\left(m_{1}+\mu_{2}\left(1-\frac{\alpha_{2}}{2}\right)+\frac{\alpha_{2}}{2} s\right) \\
& \times s \Gamma\left(p+\frac{\alpha_{2}}{2} s\right)\left(\mu_{2}\left(\frac{m_{1}^{2} C z}{\bar{\gamma}_{1} \bar{\gamma}_{2}^{(1)}}\right)^{\frac{\alpha_{2}}{2}}\right)^{-s} d s \\
& =H_{1,4}^{3,1}\left(\mu_{2}\left(\frac{m_{1}^{2} C z}{\bar{\gamma}_{1} \bar{\gamma}_{2}^{(1)}}\right)^{\frac{\alpha_{2}}{2}} \begin{array}{c}
(0,1) ;- \\
\Delta_{1} ;(1,1)
\end{array}\right) .
\end{aligned}
$$

Substituting 62 into 61, and performing in a similar way the differentiation with respect to $z$ done in (62) for 20 , (21) is attained. 


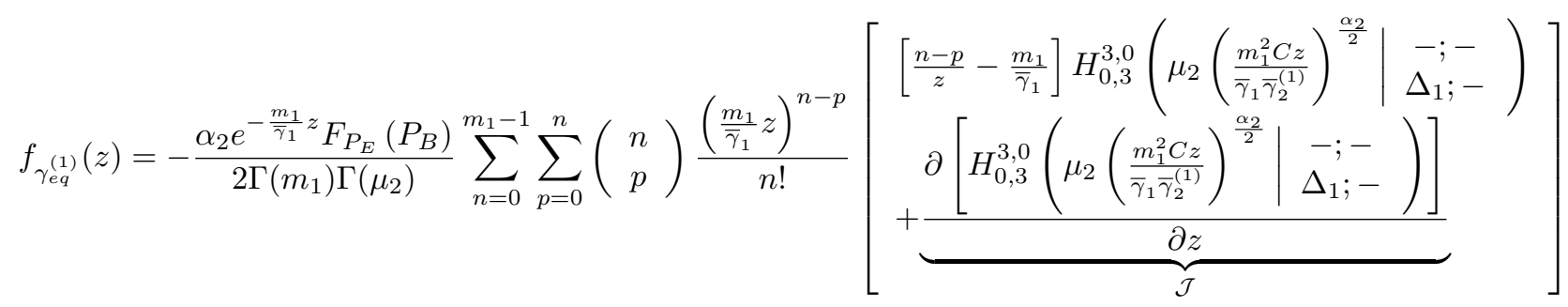

\section{APPENDiX C: ProOf OF LEMMA 1}

By plugging the definition of Fox's $H$-Function [28, Eqs. (1.2)-(1.3)] into [23, we obtain

$$
\begin{aligned}
\Xi(x) & =\frac{1}{2 \pi j} \int_{\mathcal{L}} \frac{\prod_{i=1}^{n} \Gamma\left(1-a_{i}-A_{i} s\right) \prod_{k=1}^{m} \Gamma\left(b_{k}+B_{k} s\right)}{\prod_{i=n+1}^{p} \Gamma\left(a_{i}+A_{i} s\right) \prod_{k=m+1}^{q} \Gamma\left(1-b_{k}-B_{k} s\right)} \\
& \times a^{-s} d s \int_{x}^{\infty} e^{-\lambda z} z^{-v s+\omega-1} d z
\end{aligned}
$$

where $\mathcal{L}$ is a complex contour of integration. Using [27, Eq. (8.350.2)] and after some algebraic manipulations, we obtain

$$
\int_{x}^{\infty} e^{-\lambda z} z^{-v s+\omega-1} d z=\lambda^{-\omega} \Gamma(\omega-v s, \lambda x) .
$$

Finally, by inserting (64) into (63), Lemma 1 is attained.

\section{REFERENCES}

[1] L. Jiang, H. Tian, Z. Xing, K. Wang, K. Zhang, S. Maharjan, S. Gjessing, and Y. Zhang, "Social-aware energy harvesting device-to-device communications in 5G networks," IEEE Wireless Commun., vol. 23, no. 4, pp. 20-27, August 2016.

[2] Y. Zhang, R. Yu, M. Nekovee, Y. Liu, S. Xie, and S. Gjessing, "Cognitive machine-to-machine communications: visions and potentials for the smart grid," IEEE Network, vol. 26, no. 3, pp. 6-13, May 2012.

[3] Y. Zhang, J. Ge, and E. Serpedin, "Performance analysis of a $5 \mathrm{G}$ energyconstrained downlink relaying network with non-orthogonal multiple access," IEEE Trans. Wireless Commun., vol. 16, no. 12, pp. 8333-8346, Dec 2017.

[4] M. A. Abd-Elmagid, T. A. ElBatt, and K. G. Seddik, "A generalized optimization framework for wireless powered communication networks," CoRR, vol. abs/1603.01115, 2016. [Online]. Available: http://arxiv.org/ abs/1603.01115

[5] E. Hossain and M. Hasan, "5G cellular: key enabling technologies and research challenges," IEEE Instrumentation Measurement Magazine, vol. 18, no. 3, pp. 11-21, June 2015.

[6] G. Pan, H. Lei, Y. Yuan, and Z. Ding, "Performance analysis and optimization for SWIPT wireless sensor networks," IEEE Trans. Commun. vol. 65, no. 5, pp. 2291-2302, May 2017.

[7] S. Sudevalayam and P. Kulkarni, "Energy harvesting sensor nodes: Survey and implications," IEEE Commun. Surveys Tuts., vol. 13, no. 3, pp. 443 461, Third 2011.

[8] P. Kamalinejad, C. Mahapatra, Z. Sheng, S. Mirabbasi, V. C. M. Leung, and Y. L. Guan, "Wireless energy harvesting for the internet of things," IEEE Communications Magazine, vol. 53, no. 6, pp. 102-108, June 2015

[9] J. Ye, H. Lei, Y. Liu, G. Pan, D. B. da Costa, Q. Ni, and Z. Ding, "Cooperative communications with wireless energy harvesting over Nakagami$m$ fading channels," IEEE Trans. Commun., vol. 65, no. 12, pp. 51495164, Dec 2017.

[10] D. Niyato, D. I. Kim, M. Maso, and Z. Han, "Wireless powered communication networks: Research directions and technological approaches," IEEE Wireless Commun., vol. 24, no. 6, pp. 88-97, Dec 2017.

[11] Y. Gu and S. Aïssa, "RF-based energy harvesting in decode-and-forward relaying systems: Ergodic and outage capacities," IEEE Trans. Wireless Commun., vol. 14, no. 11, pp. 6425-6434, Nov 2015.

[12] M.-K. Simon and M.-S. Alouini, Digital Communication Over Fading Channels. New York: John Wiley and Sons, 2005.

[13] M. D. Yacoub, "The $\alpha-\mu$ distribution: A physical fading model for the Stacy distribution,” IEEE Trans. Veh. Technol., vol. 56, no. 1, pp. 27-34, Jan. 2007.
[14] M. O. Hasna and M. S. Alouini, "A performance study of dual-hop transmissions with fixed gain relays," IEEE Trans. Wireless Commun., vol. 3, no. 6, pp. 1963-1968, Nov. 2004

[15] P. C. Sofotasios, M. K. Fikadu, S. Muhaidat, Q. Cui, G. K. Karagiannidis, and M. Valkama, "Full-duplex regenerative relaying and energy-efficiency optimization over generalized asymmetric fading channels," IEEE Trans. Wireless Commun., vol. 16, no. 5, pp. 3232-3251, May 2017.

[16] N. Kapucu, M. Bilim, and I. Develi, "Outage probability analysis of dualhop decode-and-forward relaying over mixed Rayleigh and generalized Gamma fading channels," Wireless Pers. Commun., vol. 71, no. 2, pp. 947-954, Jul. 2013.

[17] H. A. Suraweera, R. H. Y. Louie, Y. Li, G. K. Karagiannidis, and B. Vucetic, "Two hop amplify-and-forward transmission in mixed Rayleigh and Rician fading channels," IEEE Commun. Lett., vol. 13, no. 4, pp. 227-229, Apr. 2009.

[18] Y. Ai and M. Cheffena, "Performance analysis of hybrid-ARQ with chase combining over cooperative relay network with asymmetric fading channels," in 2016 IEEE 84th Veh. Technol. Conf. (VTC-Fall), Sep. 2016, pp. 1-6.

[19] P. Jayasinghe et al., "Performance analysis of optimal beamforming in fixed-gain AF MIMO relaying over asymmetric fading channels," IEEE Trans. Commun., vol. 62, no. 4, pp. 1201-1217, Apr. 2014.

[20] M. Babaei, U. Aygölü, and E. Basar, "BER analysis of dual-hop relaying with energy harvesting in Nakagami- $m$ fading channel," IEEE Trans. Wireless Commun., vol. 17, no. 7, pp. 4352-4361, July 2018.

[21] C. Zhong, H. A. Suraweera, G. Zheng, I. Krikidis, and Z. Zhang, "Wireless information and power transfer with Full Duplex relaying," IEEE Trans. Commun., vol. 62, no. 10, pp. 3447-3461, Oct 2014.

[22] C. Xu, M. Zheng, W. Liang, H. Yu, and Y. Liang, "End-to-end throughput maximization for underlay multi-hop cognitive radio networks with RF energy harvesting," IEEE Trans. Wireless Commun., vol. 16, no. 6, pp. 3561-3572, June 2017.

[23] J. Zhang and G. Pan, "Outage analysis of wireless-powered relaying mimo systems with non-linear energy harvesters and imperfect CSI," IEEE Access, vol. 4, pp. 7046-7053, 2016.

[24] L. Rubio, J. Reig, and N. Cardona, "Evaluation of Nakagami fading behaviour based on measurements in urban scenarios," $A E U$ - Int. J. Electron. Commun., vol. 61, pp. 135-138, Feb. 2007.

[25] K. Oliveira, A. D. O. Moraes, E. Costa, M. T. Muella, E. R. de Paula, and W. Perrella, "Validation of the $\alpha-\mu$ model of the power spectral density of gps ionospheric amplitude scintillation," Int. J. Antennas Propag., vol. 2014, pp. 1-9, Dec. 2014.

[26] A. de Oliveira Moraes et al., "On the distribution of GPS signal amplitudes during low-latitude ionospheric scintillation," GPS Solutions, vol. 17, no. 4, pp. 499-510, Oct. 2013.

[27] I. S. Gradshteyn and I. M. Ryzhik, Table of Integrals, Series, and Products: Seventh Edition. Burlington, MA: Elsevier, 2007.

[28] A. Mathai, R. K. Saxena, and H. J. Haubol, The H-Function Theory and Applications. New York: Springer, 2010.

[29] F. Yilmaz and M. S. Alouini, "Product of shifted exponential variates and outage capacity of multicarrier systems," in 2009 European Wireless Conf., May 2009, pp. 282-286.

[30] E. Illi, F. E. Bouanani, and F. Ayoub, "A performance study of a hybrid $5 \mathrm{G}$ RF/FSO transmission system," in 13th Int. Conf. Wireless Net. Mobile Commun. (WINCOM), Nov. 2017, pp. 1-7.

[31] P. K. Mittal and K. C. Gupta, "An integral involving generalized function of two variables," Proceedings of the Indian Academy of Sciences Section A, vol. 75, no. 3, pp. 117-123, Mar. 1972.

[32] I. W. Research, Mathematica Edition: version 11.3. Champaign, Illinois: Wolfram Research, Inc., 2018.

[33] J. Kang, I. Kim, and D. I. Kim, "Joint optimal mode switching and power adaptation for nonlinear energy harvesting SWIPT system over fading channel," IEEE Trans. Commun., vol. 66, no. 4, pp. 1817-1832, April 2018.

[34] L. Liu, R. Zhang, and K. Chua, "Wireless information transfer with opportunistic energy harvesting," IEEE Trans. Wireless Commun., vol. 12, no. 1, pp. 288-300, January 2013. 
[35] P. S. Bithas and P. T. Mathiopoulos, "Capacity of correlated generalized Gamma fading with dual-branch selection diversity," IEEE Trans. Veh. Technol., vol. 58, no. 9, pp. 5258-5663, Nov. 2009.

[36] I. S. Ansari, "On the Performance of Free-Space Optical Systems over Generalized Atmospheric Turbulence Channels with Pointing Errors,' Ph.D. dissertation, King Abdullah University of Science and Technology (KAUST), Thuwal, Makkah Province, Kingdom of Saudi Arabia, February 2015

[37] A. A. Kilbas and M. Saigo, H-Transforms: Theory and Applications. Boca Raton, Florida, US: CRC Press, 2004.

[38] N. T. Hai and S. B. Yakubovich, The Double Mellin-Barnes Type Integrals and their Applications to Convolution Theory. P O Box 128, Farrer Road, Singapore 9128: World Scientific Publishing Co. Pte. Ltd, 1992. 